\title{
OTIMIZAÇÃO OPERACIONAL DE TROCADORES DE CALOR EM PARALELO CONSIDERANDO EFEITOS TÉRMICOS E HIDRÁULICOS
}

\author{
R. L.SILVA, A. L. H.COSTA \\ Universidade do Estado do Rio de Janeiro, Programa de Pós-Graduação em Engenharia Química \\ E-mail para contato: rachelluiza.eng@gmail.com
}

\begin{abstract}
RESUMO - A deposição que ocorre na superfície dos tubos de trocadores de calor casco e tubo acarreta efeitos térmicos e hidráulicos durante sua operação, elevando assim os custos e comprometendo a eficiência energética da rede. Neste trabalho, os efeitos térmicos e hidráulicos são considerados simultaneamente em um sistema de trocadores em paralelo onde a escolha da abertura das válvulas de controle determina a distribuição da vazão entre os equipamentos, com impacto direto na taxa de transferência de calor recuperado. Diante deste cenário, propõe-se a utilização de uma ferramenta de programação matemática para maximizar a temperatura de saída da corrente fria, explorando a diferença da deposição entre os trocadores, sem desrespeitar a carga hidráulica disponibilizada pela bomba presente no sistema. O desempenho da rotina computacional desenvolvida é analisado através de um exemplo típico, onde percebe-se os ganhos obtidos com a otimização proposta.
\end{abstract}

\section{INTRODUÇÃO}

Redes de trocadores de calor são estruturas que permitem a transferência de calor entre correntes de processo de forma a reduzir o consumo de utilidades. Entretanto, parte do ganho obtido na recuperação de calor é perdido ao longo do tempo devido ao acúmulo de depósitos sobre as superfícies de troca térmica. Em função da importância deste problema, um grande número de trabalhos tem focado o gerenciamento da deposição visando mitigar o seu impacto do ponto de vista térmico (Bott, 1995).Entretanto, a deposição também possui um impacto hidráulico intrinsecamente associado ao problema térmico. O acúmulo dos depósitos não só reduz a efetividade dos trocadores, como também aumenta a resistência ao escoamento das correntes. Apenas recentemente, análises termo hidráulicas mais completas têm sido desenvolvidas para o gerenciamento da deposição, embora ainda com limitações (Ishiyama, 2008; Assis et al., 2013).

Neste contexto, o presente trabalho propõe a otimização da distribuição das vazões em redes de trocadores de calor compostas por equipamentos em paralelo. Busca-se determinar o perfil ótimo de abertura das válvulas ao longo do tempo capaz de garantir a minimização do impacto da deposição em termos do consumo de energia, explorando cenários onde há diferenças na quantidade de depósitos acumulados em cada equipamento. 


\section{FORMULAÇÃO MATEMÁTICA}

\subsection{Sistema Investigado}

Considera-se como objeto de investigação estruturas compostas por um conjunto de trocadores de calor organizados em ramais em paralelo, compondo uma seção de uma rede associada a uma mesma bomba responsável por suprir a carga hidráulica necessária ao escoamento. Admite-se que os trocadores de calor possuem níveis de incrustação diferentes, devido a diferenças no tempo de limpeza ou no seu histórico operacional. Do ponto de vista da instrumentação, o sistema é dotado de uma válvula de controle de vazão para cada ramal.

\subsection{Descrição das Redes de Trocadores de Calor}

A estrutura da rede de trocadores é representada por um dígrafo. Os vértices são identificados pelo índice $t \in V E T$, representando os elementos da rede: trocadores de calor $(H E)$, bomba $(P U)$, misturadores $(M X)$, divisores de corrente $(S P)$, unidades de suprimento $(P S)$ e demanda $(P D)$. As unidades de suprimento e demanda representam as correntes a montante e a jusante da rede. As arestas conectam os vértices e são identificadas pelo índice $k \in S T R$, representando as correntes da rede, frias $(C S T R \subset S T R)$ e quentes $(H S T R \subset S T R)$. Cada seção é identificada pelo índice $s \in S$ e os ramais pelo índice $r \in R$. Os ramais pertences a uma seção $s$ são agrupados nos conjuntos $R_{s}\left(r \in R_{s}\right)$.Os vértices e aresta são longo de um ramal $r$ são agrupados nos conjuntos $V E T_{r}$ e $S T R_{\mathrm{r}}\left(t \in V E T_{r}\right.$ e $\left.k \in S T R_{\mathrm{r}}\right)$. O processo avança ao longo do tempo, onde cada instante é identificado por um índice $\tau \in T I$.

\subsection{Variáveis de Otimização}

As principais variáveis da otimização são as vazões mássicas e temperaturas das correntes $\left(m_{k, \tau}\right.$ e $T_{k, \tau}$ ); as vazões e temperaturas das correntes de entrada e saída da rede nos vértices de suprimento e demanda $\left(n_{t, \tau}\right.$ e $\left.V_{t, \tau}\right)$;as cargas térmicas nos trocadores $\left(Q_{t, \tau}\right)$; os coeficientes globais de transferência

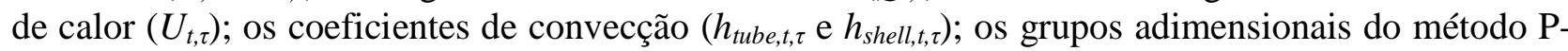
NUT: efetividade $\left(P_{t, \tau}\right)$, número de unidades de transferência $\left(N U T_{t, \tau}\right)$, e razão entre as capacidades térmicas $\left(C R_{t, \tau}\right)$; as resistências de depósito nos trocadores $\left(R_{f, t, \tau}\right)$; as taxas de deposição $\left(d R_{f, t, \tau}\right)$; as perdas de carga nas válvulas $\left(\triangle P_{V A L V E, k, \tau}\right)$; as perdas de carga nas tubulações $\left(\triangle P_{P I P E, k, \tau}\right)$; as perdas de carga nos trocadores $\left(\Delta P_{H E, t, \tau}\right)$; as perdas de carga nos ramais $\left(\Delta P_{B R, r, \tau}\right)$; as perdas de carga nas seções $\left(\triangle P_{S E C, s, \tau}\right) ;$ e as perdas de carga nas bombas $\left(\triangle P_{P U M P, t, \tau}\right)$.

\subsection{Função Objetivo}

A função objetivo é representada pela integral do consumo de energia durante a operação:

$$
\min \sum_{t \in P D} \sum_{\tau \in T I} p_{\tau} s_{\tau} C_{O P, t} n_{t, \tau} C P_{t}\left(V_{r e f, t}-V_{t, \tau}\right)
$$

onde $p_{\tau}$ são os pesos do procedimento de integração numérica, $s_{\tau}$ são os fatores de tempo presente, $C_{O P, t}$ são os custos das utilidades, $C P_{t}$ são as capacidades caloríficas das correntes de saída da rede e 
$V_{r e f, t}$ os valores de temperatura destas correntes na ausência de depósitos (referência para avaliação do impacto da deposição).

\subsection{Restrições}

Balanço de massa: A conservação de massa nos vértices é representada por:

$$
\begin{array}{ll}
\sum_{k \in S_{t}^{\text {in }}} m_{k, \tau}-\sum_{k \in S_{t}^{\text {out }}} m_{k, \tau}=0, & t \in(M X \cup S P \cup P U M P), \tau \in T I \\
\sum_{k \in S_{t}^{\text {in }}} m_{k, \tau}-\sum_{k \in S_{t}^{\text {out }}} m_{k, \tau}+n_{t, \tau}=0, & t \in(P S \cup P D), \tau \in T I \\
\sum_{k \in\left(S_{t}^{\text {in }} \cap H S T R\right)} m_{k, \tau}-\sum_{k \in\left(S_{t}^{\text {out }} \cap H S T R\right)} m_{k, \tau}=0, & t \in H E, \tau \in T I \\
\sum_{k \in\left(S_{t}^{\text {in }} \cap C S T R\right)} m_{k, \tau}-\sum_{k \in\left(S_{t}^{\text {out }} \cap C S T R\right)} m_{k, \tau}=0, & t \in H E, \tau \in T I \\
n_{t, \tau}-n_{t, \tau}^{\text {spe }}=0, \quad t \in P S, \tau \in T I &
\end{array}
$$

onde $S_{t}^{\text {in }}$ e $S_{t}^{\text {out }}$ são os conjuntos de correntes que entram e saem do vértice $t$.

Balanço de energia: Para cada um dos vértices, o balanço é descrito por:

$$
\begin{aligned}
& \sum_{k \in S_{t}^{\text {in }}} m_{k, \tau} C p_{k, \tau} T_{k, \tau}-\sum_{k \in S_{t}^{\text {out }}} m_{k, \tau} C p_{k, \tau} T_{k, \tau}=0, \quad t \in(M X \cup S P \cup P U M P), \tau \in T I \\
& \sum_{k \in S_{t}^{\text {in }}} m_{k, \tau} C p_{k, \tau} T_{k, \tau}-\sum_{k \in S_{t}^{\text {out }}} m_{k, \tau} C p_{k, \tau} T_{k, \tau}+n_{t, \tau} C P_{t} V_{t, \tau}=0, \quad t \in(P S \cup P D), \tau \in T I \\
& \quad \sum_{k \in\left(S_{t}^{\text {in }} \cap H S T R\right)} m_{k, \tau} C p_{k} T_{k, \tau}-\sum_{k \in\left(S_{t}^{\text {out }} \cap H S T R\right)} m_{k, \tau} C p_{k} T_{k, \tau}-Q_{t, \tau}=0, \quad t \in H E, \tau \in T I \\
& \sum_{k \in\left(S_{t}^{\text {in }} \cap C S T R\right)} m_{k, \tau} C p_{k, \tau} T_{k, \tau}-\sum_{k \in\left(S_{t}^{\text {out }} \cap C S T R\right)} m_{k, \tau} C p_{k} T_{k, \tau}+Q_{t, \tau}=0, \quad t \in H E, \tau \in T I \\
& V_{t, \tau}-V_{t, \tau}^{\text {spe }}=0, \quad t \in P S, \tau \in T I
\end{aligned}
$$




$$
\begin{aligned}
& \sum_{k \in S_{t}^{i n}} T_{k, \tau}-\sum_{k \in S_{t}^{\text {out }}} T_{k, \tau}=0, \quad t \in P U M P, \tau \in T I \\
& T_{k, \tau}-T_{k^{\prime}, \tau}=0, \quad t \in S P, k \in S_{t}^{\text {in }}, k^{\prime} \in S_{t}^{\text {out }}, \tau \in T I
\end{aligned}
$$

Equações de transferência de calor: As equações são baseadas no método P-NUT (Shah e Sekulic, 2003):

$$
\begin{gathered}
P_{t, \tau}-2\left\{1+C R_{t, \tau}+\left(1+C R_{t, \tau}^{2}\right)^{0.5} \frac{1+\exp \left[-N U T_{t, \tau}\left(1+C R_{t, \tau}^{2}\right)^{0.5}\right]}{1-\exp \left[-N U T_{t, \tau}\left(1+C R_{t, \tau}^{2}\right)^{0.5}\right]}\right\}^{-1} \\
=0, \quad t \epsilon H E, \tau \epsilon T I \\
N U T_{t, \tau} m_{k, \tau} C p_{k}-U_{t, \tau} A_{t}=0, \quad t \epsilon H E, k \epsilon\left(H S T R \cap S_{t}^{i n}\right), \tau \epsilon T I \\
C R_{t, \tau} m_{k, \tau} C p_{k, \tau}-m_{k^{\prime}, \tau} C p_{k^{\prime}}=0, \\
t \epsilon H E, k \epsilon\left(C S T R \cap S_{t}^{i n}\right), k^{\prime} \epsilon\left(H S T R \cap S_{t}^{i n}\right), \tau \epsilon T I
\end{gathered}
$$

onde $A_{t}$ é a área de troca térmica, os subscritos $h$ e $c$ correspondem a correntes quentes e frias respectivamente e $i$ e $o$ representam correntes de entrada e saída. Na Equação (14), considera-se, por hipótese, que os trocadores possuem múltiplos passes nos tubos.

O coeficiente global de transferência de calor é descrito tendo como referência o diâmetro interno:

$$
\frac{1}{U_{t, \tau}}-\left(\frac{1}{h_{\text {shell }, t, \tau}}\left(\frac{D_{i, t}}{D_{e, t}}\right)+\frac{D_{i, t} \log \left(D_{e, t} / D_{i, t}\right)}{2 k_{w, t}}+\frac{1}{h_{t u b e, f, t, \tau}}+R_{f, t, \tau}\right)=0, \quad t \in H E, \tau \in T I
$$

A relação entre os coeficientes de convecção sujo e limpo, e a relação entre os coeficientes de convecção interno e externo e a vazão são expressas por:

$$
\begin{aligned}
& h_{\text {tube }, f, t, \tau}-h_{\text {tube }, t, \tau}\left(D_{i, t} / D_{i, f, t, \tau}\right)^{1.8}=0, \quad t \in H E, \tau \in T I \\
& h_{\text {tube }, t, \tau}-h_{\text {tube }, t}^{\text {base }}\left[\frac{c_{t} m_{k, \tau}+\left(1-c_{t}\right) m_{k^{\prime}, \tau}}{m_{\text {tube, }}^{\text {base }}}\right]^{0.8} \\
& =0, t \epsilon H E, k \epsilon\left(C S T R \cap S_{t}^{\text {in }}\right), k^{\prime} \in\left(H S T R \cap S_{t}^{\text {in }}\right), \tau \in T I
\end{aligned}
$$




$$
\begin{aligned}
h_{\text {shell }, t, \tau}-h_{\text {shell }, t}^{\text {base }} & {\left[\frac{\left(1-c_{t}\right) m_{k, \tau}+c_{t} m_{k^{\prime}, \tau}}{m_{\text {shell }, t}^{\text {base }}}\right]^{0.6} } \\
= & 0, t \in H E, k \epsilon\left(\operatorname{CSTR} \cap S_{t}^{\text {in }}\right), k^{\prime} \epsilon\left(H S T R \cap S_{t}^{\text {in }}\right), \tau \in T I
\end{aligned}
$$

onde $D_{i, f}$ é o diâmetro interno do tubo com incrustação, $m_{\text {tube,t }}^{\text {base }}, m_{\text {shell,t }}^{\text {base }}, h_{\text {tube,t }}^{\text {base }}$ e $h_{\text {shell,t }}$ base ${ }_{\text {são }}$ valores previamente conhecidos de vazão mássica e coeficiente de convecção para um caso base e $c_{t}$ é um parâmetro indicativo da posição dos fluidos dentro do trocador de calor $(=1$, corrente fria escoa nos tubos, $=0$, corrente quente escoa no casco).

Modelagem da deposição: Para descrever o comportamento da deposição, foi adotado o modelo de Ebert-Panchal modificado (Panchal et al., 1997), descrito pelo conjunto de restrições a seguir:

$$
\begin{aligned}
& d R_{f, t, \tau}=0,5\left(d R_{f 1, t, \tau}+d R_{f 2, t, \tau}\right) \quad t \in H E, \tau \epsilon T I \\
& d R_{f 1, t, \tau} \geq \alpha\left(R e_{t, \tau}^{-0.66}\right) P r_{t}^{0.33} \exp \left(-E_{a} R^{-1} T_{f i l m 1, t, \tau}^{-1}\right)-\gamma \tau_{w, t, \tau} \quad t \epsilon H E, \tau \epsilon T I \\
& d R_{f 1, t, \tau} \geq 0 \quad t \epsilon H E, \tau \epsilon T I \\
& d R_{f 2, t, \tau} \geq \alpha\left(\operatorname{Re}_{t, \tau}^{-0.66}\right) P r_{t}^{0.33} \exp \left(-E_{a} R^{-1} T_{f i l m 2, t, \tau}^{-1}\right)-\gamma \tau_{w, t, \tau} \quad t \in H E, \tau \in T I \\
& d R_{f 2, t, \tau} \geq 0 \quad t \epsilon H E, \tau \epsilon T I
\end{aligned}
$$

onde $\alpha, E_{a}$, e $\gamma$ são parâmetros empíricos dos modelos de taxa de deposição, $R e_{t, \tau}$ é o número de Reynolds, $P r_{t}$ é o número de Prandtl, $R$ é a constante universal dos gases, $T_{\text {film }}$ é a temperatura de filme no interior do trocador e $\tau_{w, t, \tau}$ é a tensão de cisalhamento. Os subscritos 1 e 2indicam as extremidades do trocador.

Balanço hidráulico: Considera-se que o modelo hidráulico é aplicado apenas para a corrente fria, que escoa no lado dos tubos. As perdas de carga nas tubulações, nos trocadores, bombas e válvulas são expressas por:

$$
\begin{aligned}
& \Delta P_{P I P E, k, \tau}-\frac{f_{\text {pipe }, k, \tau} \rho_{k} L_{\text {pipek }} v_{\text {pipe }, k, \tau}^{2}}{2 d_{\text {pipe }, k}}=0, k \epsilon C S T R, \tau \epsilon T I \\
& \Delta P_{H E, t, \tau}-\frac{f_{\text {tube,t }} \rho_{t} N_{p t, t} L_{\text {tube, },} v_{\text {tube }, t, \tau}^{2}}{2 d_{i, f, t, \tau}}+K_{t} \rho_{t} N_{p t, t} \frac{v_{t, \tau}^{2}}{2}=0, \quad t \epsilon H E, \tau \epsilon T I
\end{aligned}
$$




$$
\begin{aligned}
& \Delta P_{P U M P, t, \tau}-\left(-a_{t} q_{k, \tau}^{2}+b_{t} q_{k, \tau}+c_{t}\right) \rho_{k} g=0, \quad t \in P U, k \in C S T R, \tau \in T I \\
& \Delta P_{V A L V E, k, \tau}-\rho_{k}\left(\frac{q_{k, \tau}}{R^{x_{k, \tau}-1} C v_{k}}\right)^{2}=0, k \in C S T R, \tau \in T I, r \in R
\end{aligned}
$$

onde os subscritos pipe e tube se referem às tubulações e tubos dos trocadores, respectivamente, $f_{\text {pipe, }, k} \mathrm{e}$ $f_{\text {tube, } t}$ são os valores de fator de atrito, $L_{p i p e, k}$ e $L_{\text {tube }, k}$ são os comprimentos, $v_{p i p e, k, \tau}$ e $v_{\text {tube, }, \tau}$ são as velocidades de escoamento, $d_{p i p e, k}$ são os diâmetros das tubulações, $N_{p t, t}$ são os números de passes nos tubos dos trocadores, $q_{k, \tau}$ são as vazões volumétricas, $R^{x-1}$ são parâmetros da equação característica da válvula (assumindo comportamento igual percentagem) e $x_{k, \tau}$ são as posições dos acionadores das válvulas.

A queda de pressão no ramal é a soma das quedas de pressão nas tubulações, nos trocadores e na válvula pertencentes ao ramal:

$$
\begin{gathered}
\Delta \mathrm{P}_{B R, r, \tau}-\sum_{k \in S T R_{r}} \Delta \mathrm{P}_{\mathrm{PIPE}, \mathrm{k}, \tau}-\sum_{t \in\left(H E \cap V E T_{r}\right)} \Delta P_{H E, t, \tau}-\sum_{k \in\left(V A L V \cap S T R_{r}\right)} \Delta P_{V A L V E, k, \tau} \\
=0, \quad t \in H E, k \in S T R, \tau \in T I, r \in R
\end{gathered}
$$

onde as correntes que possuem válvulas estão contidas no conjunto $V A L V$ ( $k \epsilon V A L V \subset S T R)$.

Uma vez que a queda de pressão nos ramais em paralelo de uma seção são iguais, tem-se:

$$
\Delta P_{S E C, s, \tau}-\Delta \mathrm{P}_{\mathrm{BR}, \mathrm{r}, \tau}=0, \quad s \in S, \quad r \in R_{S}, \quad \tau \in T I
$$

onde o subscrito $S E C$ se refere as seções.

A queda de pressão na seção é suprida pela bomba, assim:

$$
\Delta P_{S E C, s, \tau}-\Delta P_{\mathrm{PUMP}, \mathrm{t}, \tau}=0, \quad s \in S, \quad t \in\left(P U \cap V E T_{s}\right), \quad \tau \in T I
$$

Limites das variáveis: Os limites das variáveis vazão e temperatura são necessários para que estas não ultrapassem especificações operacionais desejadas e são descritos nas Equações 33 e 34.

$$
\begin{aligned}
& m_{k}^{L B} \leq m_{k} \leq m_{k}^{U B}, k \in S P E m \\
& T_{k}^{L B} \leq T_{k} \leq T_{k}^{U B}, k \in S P E T
\end{aligned}
$$

onde SPEm e SPET são os conjuntos das especificações operacionais. 


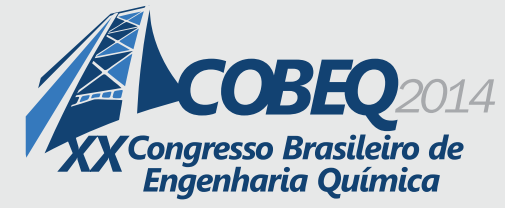

\section{RESULTADOS}

O caso em estudo é composto por dois trocadores em paralelo, projetados para aquecer uma corrente fria, como ilustra a Figura 1. A otimização foi implementada no software GAMS, resultando em um problema de programação não linear (NLP), resolvido através do solver CONOPT. O tempo considerado para o horizonte operacional foi de 53 semanas. A corrente fria tem uma vazão de 310 $\mathrm{kg} / \mathrm{s}$, temperatura de $157{ }^{\circ} \mathrm{C}$, massa especifica de $819 \mathrm{~kg} / \mathrm{m}^{3}$, viscosidade de $0,0013 \mathrm{~Pa} . \mathrm{s}$, condutividade térmica de $0,1 \mathrm{~W} / \mathrm{m} . \mathrm{K}$ e capacidade calorífica de $2378 \mathrm{~J} / \mathrm{kg} . \mathrm{K}$. A corrente quente tem vazão de $176 \mathrm{~kg} / \mathrm{s}$, temperatura de $214^{\circ} \mathrm{C}$ e capacidade calorífica de $2476 \mathrm{~J} / \mathrm{kg} . \mathrm{K}$. Os trocadores são compostos por 1520 tubos de $3 / 4$ in e $6,1 \mathrm{~m}$ de comprimento, distribuídos ao longo de 2 passes. No início um dos trocadores parte com resistência de deposito inicial igual a $0,00098 \mathrm{~m}^{2} \mathrm{~K} / \mathrm{W}$ e o outro está limpo. As válvulas em cada corrente são idênticas. Os parâmetros utilizados para a Equação $22 \mathrm{e}$ 24 são $\alpha=127,7, E_{a}=76000 \mathrm{~J} / \mathrm{mol}$ e $\gamma$ igual a $3,44 \cdot 10^{-15}$. Considera-se que o custo da utilidade quente é unitário e o custo da utilidade fria desprezível, assim, a função objetivo se torna a minimização da perda de energia devido à deposição.

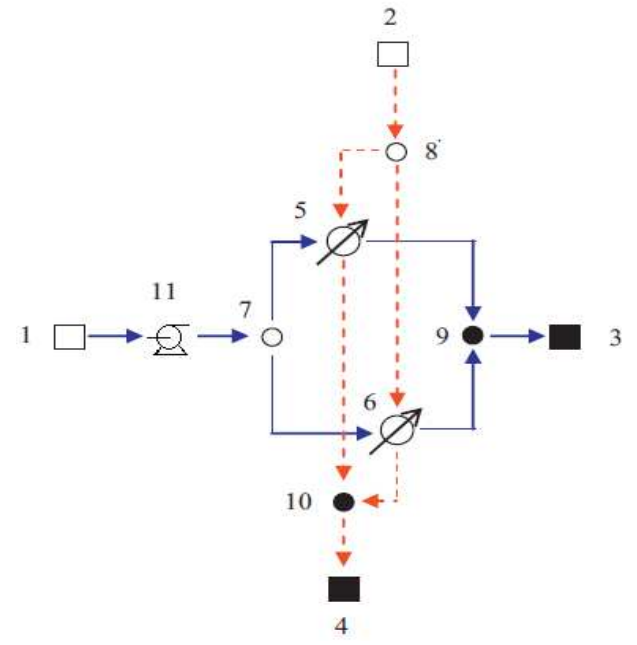

Figura 1- Esquema da rede de trocadores de calor

Visando avaliar o desempenho do procedimento de otimização, este foi confrontado com uma política operacional proposta por Ishiyama et al. (2008), onde as vazões são mantidas equivalentes nos dois ramais ao longo do tempo. Estes autores propõem esta alternativa de forma a evitar o desbalanceamento em função do comportamento hidráulico do sistema, o que seria prejudicial à transferência de calor ("thermochanneling").

Aplicando a abordagem proposta em Ishiyama et al. (2008), o consumo de energia associado à deposição foi de 0,157 PJ. O resultado obtido através da otimização alcançou um consumo de energia de 0,147 PJ, i.e., 6,4\% menor. A Figura 2 apresenta a evolução da vazão e o posicionamento das válvulas ao longo da campanha operacional investigada. Neste caso, é possível observar que o fluxo é 


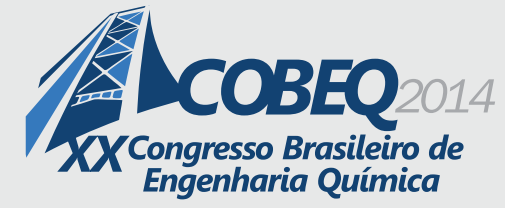

19 a 22 de outubro de 2014

Florianópolis/SC

inicialmente desviado para o trocador menos sujo, porém ao longo do tempo este tende a ser redistribuído de maneira mais uniforme.

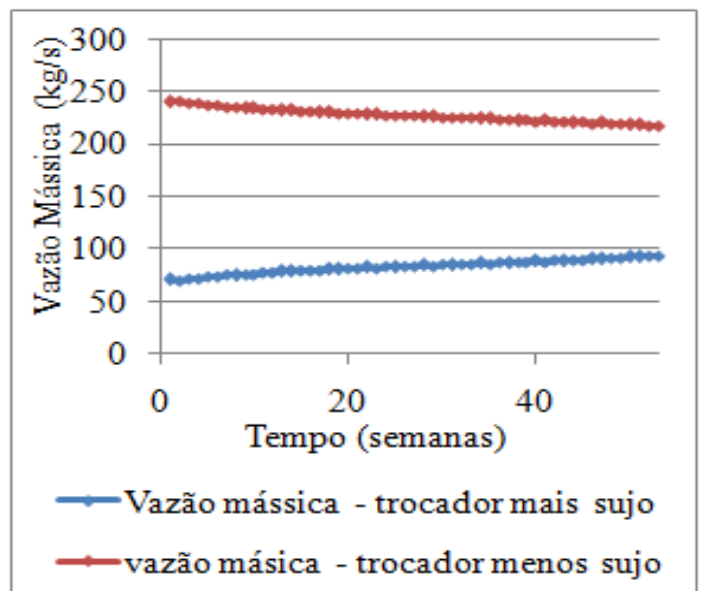

(a)

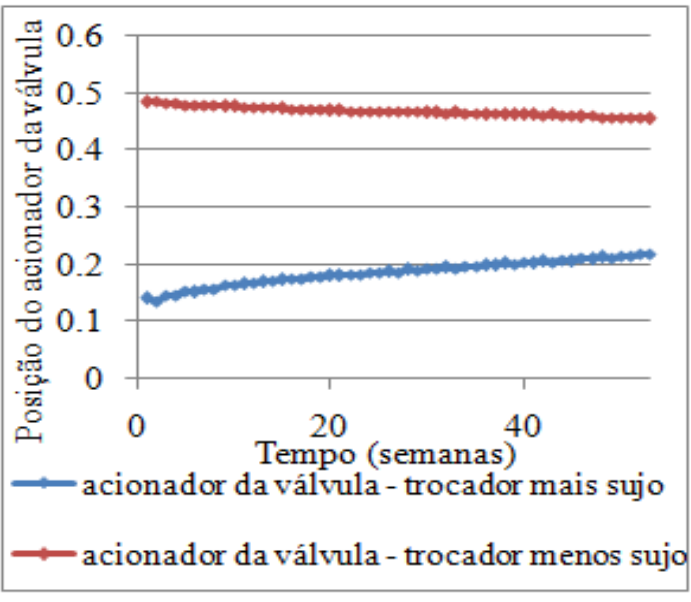

(b)

Figura 2- (a) Perfil da vazão mássica ao longo do tempo e (b) Perfil do acionador da válvula ao longo do tempo

\section{CONCLUSÃO}

Com a formulação apresentada neste trabalho, foi possível executar a otimização termohidráulica da distribuição das vazões ao longo de uma rede de trocadores de calor, de forma a diminuir o impacto resultante da deposição. O resultado da otimização apresenta menor perda de energia do que a proposição estabelecida pela literatura, pois torna-se assim possível direcionar o fluxo de forma a otimizar o custo, respeitando o balanço hidráulico.

\section{REFERÊNCIAS}

ASSIS, B.C.G.; GONÇALVES C.O.; LIPORACE F.S.; OLIVEIRA S.G.; QUEIROZ E.M.; PESSOA L.P.; COSTA A.L.H. Constrained thermohydraulic optimization of the flow rate distribution in crude preheat trains. Chem. Eng. Res.Des.,v. 91, p 1517-1526, 2013.

BOTT, T. R., Fouling of Heat Exchangers. Elsevier, 1995.

ISHIYAMA, E. M.; PATERSON, W.R., WILSON, D.I. Thermo-hydraulic channelling in parallel heat exchangers subject to fouling. Chem. Eng. Sci., v. 63, p. 3400-3410, 2008.

PANCHAL, C. B.; KURU, W. C.; LIAO, C. F.; EBERT, W. A.; PALEN, J. W. Threshold Conditions for Crude Oil Fouling. In: International Conference on Understanding Heat Exchanger Fouling and its Mitigation, 1997.

SHAH, R. K., SEKULIC, D. P., Fundamentals of Heat Exchanger Design. Hoboken, New Jersey: John Wiley and Sons, 2003. 